\title{
Glycolic Acid, Lactic Acid, Mandelic Acid, Salicylic Acid, Citric Acid, Gluconolactone: Skin Exfoliators in Combination Therapy of Acne Vulgaris
}

\author{
Vishal Saxena ${ }^{1 *}$, Krishana Yadav ${ }^{2}$ \\ ${ }^{1}$ Research Scholar, Department of Pharmacy, B. N. University, Udaipur, India \\ ${ }^{2}$ Research Scholar, Department of Pharmacy, The Maharaja Sayajirao University of Baroda, Vadodara, India \\ *Corresponding author: saxena.visha11984@outlook.com
}

\begin{abstract}
Facial acne are a recurring issue in most of the population and can cause disturbing skin issues. A combination of Alpha-hydroxy acids, Beta-hydroxy-acids and Poly-hydroxy acids has become popular for treating acne vulgaris in last few years. The main objective of this research study is to evaluate the efficacy $\&$ safety of combination of these acids in the treatment of acne vulgaris.

The study was conducted on 12 volunteers having mild to moderate to severe acne. They were divided into 3 groups according to the severity of their facial acne. Members of each group were treated with a series of $30 \%$ AHA, $2 \%$ BHA and $7 \%$ PHA. The improvement in their acne condition as well as skin texture was assessed over a period of 6 weeks.
\end{abstract}

Keywords: Citric acid, Glycolic acid, Gluconolactone, Lactic acid, Mandelic acid, Salicylic acid.

\section{Introduction}

Acne vulgaris is one of the most persistent form of skin disease across all age groups. While in some individuals, acne is a rather minor skin issue, some face a series of very aggravated acne that even leaves behind scars [1]. Hence, a solution to that not only treats acne, but also reduces the acne scars is a prime necessity. Application of direct acids has been used for several years now with proven benefits in reduction of acne vulgaris. The combination of these acids further helps in restructuring of collagen \& surface properties of skin, which in turn results in reduction of acne scars.

Alpha-hydroxy acids (AHA), Beta-hydroxy acids (BHA) and Poly-hydroxy acids (PHA) are widely used direct acid forms. While AHA and BHA are common direct acids and have been in existence for several years, PHA are a newer generation of AHA and gaining popularity for their non-irritating \& hydration properties while exfoliating stratum corneum.

Glycolic acid is the smallest AHA (with molecular weight of $76 \mathrm{~g} / \mathrm{mol}$ ) and has maximum skin penetration, thus having maximum impact on the cell turn-over rate. It also is a hydrophilic direct acid. Similarly, Lactic acid \& Mandelic acid belong to AHA family but owing to their larger molecular weight have slower skin penetration. But Lactic \& Mandelic acids are known form of skin exfoliants without the irritation generally associated with Glycolic acid [2]. Salicylic acid is a oil-soluble form of direct acid and thus is best suited for its activity on acne lesions [3]. Lastly, PHAs are a newer generation of direct acids. Most commonly used PHAs are Gluconolactone \& Lactobionic acid and they are now commonly used for exfoliating sensitive and/or dry skin because of their milder exfoliation properties along with skin hydration [4].

The present research work is focused on the determination of efficacy of these acids in combination for treatment of acne vulgaris and acne scars.

\section{Method}

The present research was conducted on 12 volunteers having mild to severe acne condition. The 12 volunteers comprised of 5 male and 7 female and were divided into 3 groups on the basis of acne scoring. Acne scoring was done by visual inspection and categorized according to the severity of the acne.

The treatment was for an average of 6 weeks and comprised of series of applications every week with direct acid composition of $16 \%$ Glycolic acid, $8 \%$ Lactic acid, 5\% Mandelic acid \& 1\% Citric acid, 7\% Gluconolactone and 2\% Salicylic acid delivered through a gum-based water gel [5].

The above gel was topically applied once every week on each volunteer for a total duration of 6 weeks. The application time for one treatment was 10 minutes. The gel was applied on face excluding the eye and lip area.

The 6-week monitoring of each volunteer showed a visible reduction in acne and acne caused scars without any side effects. The acne starts disappearing after 3 weeks (3 applications) and acne scars appear to lighten after 4 weeks (4 applications) in all the 12 volunteers. 


\section{Discussion and Conclusion}

During the applications, the volunteers were asked to follow all possible precautions that can hamper the treatment results. Volunteers were informed about possible side effects of sunexposure and were advised to use sun protection creams every day during the treatment period and also informed to stay inhouse for at least 24 hours after the treatment day.

The AHA combination used in the study has shown significantly visible results on reduction of acne vulgaris and skin complexion. Of all AHAs, Glycolic acid has the deepest \& fastest skin penetration and has most impact on the cell turnover rate. Typical skin cell turn-over rate is $4-5$ weeks but topical application of Glycolic acid has shown to decrease this cycle, making Glycolic acid one of the most preferred skin exfoliator. These properties of Glycolic acid have a desirable effect on acne vulgaris. While there are several studies with Glycolic acid concentration as high as $70 \%$, at a concentration of $15 \%$, Glycolic acid is shown to be completely tolerable even for at-home applications.

As shown in figure 1, volunteers have seen a visible improvement after 3 weeks of treatment. BHAs are lipophilic direct acids forms and work by dissolving sebum, impurities and hence prevent the re-occurrence of the accumulation of the oil in the pores. Lastly, PHAs help in exfoliation of the stratum corneum while also providing hydration, which in turn counters the drying effects of AHAs and BHAs present in the composition.

The combination of direct acids (AHA BHA and PHA) has shown considerable effects on acne vulgaris and acne caused scars, without any side effects.
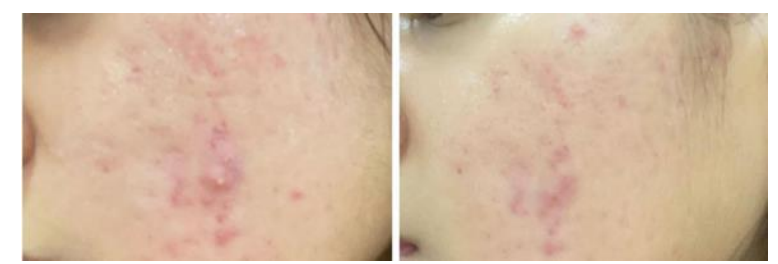

Fig. 1. Effect of AHA BHA PHA combination on skin after 3 weeks

\section{References}

[1] Ak M. "A comprehensive review of acne vulgaris". J Clin Pharm. 1:17$45,2019$.

[2] Tang SC, Yang JH. "Dual effects of alpha-hydroxy acids on the skin". Molecules. 23(4):863 April 2018.

[3] Alba MN, Gerenutti M, Yoshida VM, Grotto D. "Clinical comparison of salicylic acid peel and LED-Laser phototherapy for the treatment of Acne vulgaris in teenagers". Journal of Cosmetic and Laser Therapy. 2;19(1):49-53, January 2017.

[4] Green BA, Wildnauer RH, Edison BL. "Polyhydroxy acids (PHAs) provide conditioning effects to skin without increasing sensitivity to UV light". Amer Acad of Derm Poster Exhibit. February 2002.

[5] Wang C. M, Huang C. L, Sindy Hu C. T, Chan H. L, "The effect of glycolic acid on the treatment of acne in Asian skin" Dermatologic surgery. 23(1):23-9. Jan. 